\title{
The optimal online control of the instantaneous power and the multiphase source's current
}

\author{
M. SIWCZYŃSKI and K. HAWRON* \\ Faculty of Electrical and Computer Engineering, Institute of Electrical Engineering and Computer Science, \\ Cracow University of Technology, 24 Warszawska St., 31-155 Cracow, Poland
}

\begin{abstract}
The paper presents the new optimal real-time control algorithm of the power source. The minimum of the square-instantaneous current was assumed as an optimal criterion, with an additional constraint on source instantaneous power. The mathematical model of a multiphase source was applied as a voltage-current convolution in the discrete time domain. The resulting control algorithm was the recursive digital filter with infinite recursion.
\end{abstract}

Key words: power source, real-time control, optimum, operators.

\section{Introduction}

The paper attempts to solve new problems of the electrical source optimal real-time control. In previous papers, optimal solutions were achieved i.e. for instantaneous power exceed criterion $[1,2]$, for minimum energy losses $[3,4]$, with usage of the similarity principle [5], etc.

This article is a significant generalization of previous studies for single-phase systems [6-8] and for three-phase systems $[9,10]$. Those studies have been unsuccessful in the research for mathematical expressions for the so-called "inactive powers", while in this paper, a much more efficient approach based on optimization methods is applied.

In this paper, the two alternative cost functions were assumed as the optimal criterion: the achievement of the maximum instantaneous power or the achievement of the minimum instantaneous source's current ABS value with the given instantaneous source power. A mathematical model of multiphase power source in the discrete time domain (Fig. 1) was applied.

The source's voltage-current equation in this model is:

$$
\mathbf{u}_{n}=\mathbf{e}_{n}-\sum_{m=1}^{\infty} \mathbf{z}_{m} \mathbf{i}_{n-m}
$$

where: $\mathbf{u}_{n}=\operatorname{col}_{\alpha}\left[u_{n}^{\alpha}\right], \mathbf{e}_{n}=\operatorname{col}_{\alpha}\left[e_{n}^{\alpha}\right], \mathbf{i}_{n}=\operatorname{col}_{\alpha}\left[i_{n}^{\alpha}\right]-$ the column vectors of: the terminal phase voltage, source voltage and source output current; $\alpha=0,1, \ldots, M-1$ - the line (phase) number; $n=0,1,2, \ldots-$ the voltage and current sample's number; $\mathbf{z}_{m}=$ mat $_{\alpha, \beta}\left[z_{m}^{\alpha, \beta}\right]-$ the square matrix of the impulse responses of source's internal impedance operator; $\alpha, \beta=0,1, \ldots, M-1$

*e-mail: konhawpk@gmail.com

Manuscript submitted 2016-10-25, revised 2017-02-27, initially accepted for publication 2017-03-31, published in December 2017.

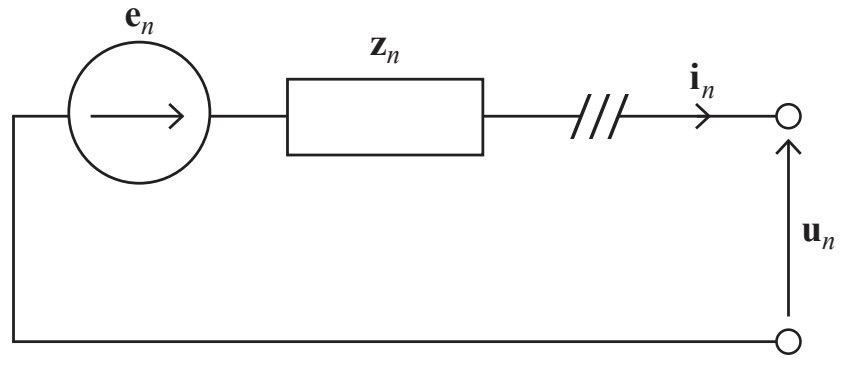

Fig. 1. A multiphase electrical power source

- the number of phase. This means that the source model assumed is a linear, time-invariant, i.e. the convolutional inner impedance operator. In particular, it is the discrete time convolution.

In the online optimal control system, the transition from the $n-1$ state to the $n$ state takes place so as to meet the given quality criteria. Two alternate criteria are assumed:

- the maximum of instantaneous power criteria (called $p_{n}^{M A X}$ algorithm), i.e.:

$$
\mathbf{i}_{n}^{T} \mathbf{u}_{n} \rightarrow M A X
$$

where $T$ means function transposition,

- the minimum of instantaneous current ABS value at a given instantaneous source power $p_{n}$, called $\left|\mathbf{i}_{n}\right|^{M I N}$ algorithm:

$$
\begin{aligned}
& \mathbf{i}_{n}^{T} \mathbf{i}_{n} \rightarrow M I N \\
& \mathbf{i}_{n}^{T} \mathbf{u}_{n}-p_{n}=0 .
\end{aligned}
$$

In order to solve these tasks, (1) is transformed in the form of recursive digital filter with infinite recursion:

$$
\mathbf{u}_{n}=\mathbf{v}_{n}-\mathbf{z}_{0} \mathbf{i}_{n}
$$


where:

$$
\mathbf{v}_{n}=\mathbf{e}_{n}-\sum_{m=1}^{\infty} \mathbf{z}_{m} \mathbf{i}_{n-m}
$$

In expressions (2) and (3) the vector of sample's sequence $\left\{\mathbf{v}_{n}\right\}$ and the matrix $\mathbf{z}_{0}$ will be treated as given values, while the vector $\left\{\mathbf{i}_{n}\right\}$ of sample's sequence will be sought.

Assumed control algorithms take the following forms: $-p_{n}^{M A X}$ algorithm:

$$
\begin{aligned}
\mathbf{i}_{n}^{T} \mathbf{u}_{n} & =\mathbf{i}_{n}^{T}\left(\mathbf{v}_{n}-\mathbf{z}_{0} \mathbf{i}_{n}\right)=\mathbf{i}_{n}^{T} \mathbf{v}_{n}-\mathbf{i}_{n}^{T} \mathbf{z}_{0} \mathbf{i}_{n}= \\
& =\mathbf{i}_{n}^{T} \mathbf{v}_{n}-\mathbf{i}_{n}^{T} \mathbf{r}_{0} \mathbf{i}_{n} \rightarrow M A X
\end{aligned}
$$

or equivalently:

$$
\mathbf{i}_{n}^{T} \mathbf{r}_{0} \mathbf{i}_{n}-\mathbf{i}_{n}^{T} \mathbf{v}_{n} \rightarrow M I N
$$

where:

$$
\mathbf{r}_{0}=\frac{1}{2}\left(\mathbf{z}_{0}+\mathbf{z}_{0}^{T}\right)
$$

is positive defined, time-invariant matrix; $-\left.\left|\mathbf{i}_{n}\right|\right|^{M I N}$ algorithm:

$$
\begin{aligned}
& \mathbf{i}_{n}^{T} \mathbf{i}_{n} \rightarrow M I N \\
& \mathbf{i}_{n}^{T}\left(\mathbf{v}_{n}-\mathbf{r}_{0} \mathbf{i}_{n}\right)-p_{n}=0,
\end{aligned}
$$

or using Lagrange's factor:

$$
\mathbf{i}_{n}^{T}\left(\mathbf{1}+\lambda \mathbf{r}_{0}\right) \mathbf{i}_{n}-\lambda \mathbf{v}_{n}^{T} \mathbf{i}_{n} \rightarrow M I N
$$

where $\mathbf{1}$ is the identity matrix

The solution of both optimization tasks, at a given $\mathbf{e}_{n}$ and $\mathbf{z}_{n}$ for $n=0,1,2, \ldots$ is the $\mathbf{i}_{n}$ sequence as a function of $\mathbf{v}_{n}$, this in turn is function of $\mathbf{i}_{n-1}, \mathbf{i}_{n-2}, \ldots, \mathbf{i}_{1}, \mathbf{i}_{0}$. Thus, the solutions of those tasks should have form of a theoretically infinite recursive digital filter.

\section{The solution of the optimal tasks for the single phase source}

For the single phase source its voltage-current equation takes the simpler (scalar) form:

$$
\begin{aligned}
u_{n} & =e_{n}-\sum_{m=0}^{\infty} z_{m} i_{n-m}=e_{n}-\sum_{m=1}^{\infty} z_{m} i_{n-m}-z_{0} i_{n}= \\
& =v_{n}-z_{0} i_{n}
\end{aligned}
$$

where:

$$
v_{n}=e_{n}-\sum_{m=1}^{\infty} z_{m} i_{n-m} .
$$

The $p_{n}^{M A X}$ task has the form:

$$
i_{n}\left(v_{n}-z_{0} i_{n}\right) \rightarrow M A X
$$

and the $\left[\left(i_{n}\right)^{2}\right]^{M I N}$ task has the form:

$$
\begin{aligned}
& \left(i_{n}\right)^{2} \rightarrow M I N \\
& i_{n}\left(v_{n}-z_{0} i_{n}\right)=p_{n}
\end{aligned}
$$

or yet another form using Lagrange's factor:

$$
\left(1+\lambda z_{0}\right)\left(i_{n}\right)^{2}-v_{n} i_{n} \rightarrow M I N
$$

In order to solve these tasks the discrete time index is skipped in (7) and (8), therefore the $p_{n}^{M A X}$ takes form:

$$
v i-r_{0} i^{2} \rightarrow M A X
$$

where $r_{0}=z_{0}$, and the $\left(i^{2}\right)^{M I N}$ takes form:

$$
\left(1+\lambda r_{0}\right) i^{2}-v i \rightarrow M I N .
$$

The solving equations for (10) and (11) tasks take form:

$$
v-2 r_{0} i=0
$$

thus:

and:

$$
i \equiv i^{d}=\frac{v}{2 r_{0}}
$$

$$
\left(1+\lambda r_{0}\right) i=\frac{1}{2} \lambda v
$$

thus:

$$
i \equiv i^{o p t}=\Lambda v
$$

where $\Lambda$ is an undetermined real scalar. The obtained currents $i^{d}$ and $i^{\text {opt }}$ will be called adjustment current and optimal current respectively. Using these currents in the source's balance condition:

$$
r_{0} i^{2}-v i+p=0
$$

the maximum power is obtained:

$$
p^{M A X}=\frac{v^{2}}{4 r_{0}},
$$

and the equation which allows to calculate the $\Lambda$ factor takes form:

$$
r_{0} v^{2} \Lambda^{2}-v^{2} \Lambda+p=0 .
$$

This quadratic equation can be formed as follows:

$$
r_{0} \Lambda^{2}-\Lambda+\frac{1}{4 r_{0}} x=0
$$

where $x=\frac{p}{p^{M A X}}$ is the so-called fraction of the source's load. The one and only allowed solution for the quadratic equation is:

$$
i=i^{o p t}=\frac{v}{2 r_{0}}(1-\sqrt{1-x})=i^{d}(1-\sqrt{1-x}) .
$$


The same result can be obtained using the source's balance condition:

$$
r_{0} i^{2}-v i+p=0
$$

which takes form:

$$
\frac{r_{0}}{v} i^{2}-i+\frac{v}{4 r_{0}} x=0
$$

and solving it with respect to $i$ yields:

$$
i=\frac{v}{2 r_{0}}(1-\sqrt{1-x})=i^{d}(1-\sqrt{1-x}) .
$$

Thus, the following theorem of the single phase source has been proved: the current that delivers the given instantaneous power from the source has the minimum or the maximum instantaneous-square value.

The $\left[\left(i_{n}\right)^{2}\right]^{M I N}$ algorithm works recursively, i.e. $\left(i_{0}, i_{1}, i_{2}, \ldots\right.$, $\left.i_{n-1}\right) \rightarrow i_{n}$ according to the scheme:

$$
\begin{aligned}
& \left(i_{n}\right)^{2} \longrightarrow \text { MIN } \\
& i_{n} u_{n}=p_{n}
\end{aligned}
$$

and is solved by the following sequences of operations:

$$
\begin{gathered}
u_{n}=e_{n}-\sum_{m=1}^{\infty} z_{m} i_{n-m}, \\
i_{n}^{d}=\frac{v_{n}}{2 r_{0}}
\end{gathered}
$$

where $r_{0}=z_{0}$,

$$
\begin{gathered}
p_{n}^{M A X}=\frac{\left(v_{n}\right)^{2}}{4 r_{0}}, \\
x_{n}=\frac{p_{n}}{p_{n}^{M A X}}, \\
i_{n}= \begin{cases}i_{n}^{d} \sqrt{1-x_{n}} & \text { for } x_{n}<1 \\
i_{n}^{d} & \text { for } x_{n}>1\end{cases}
\end{gathered}
$$

where $n=0,1,2, \ldots$ is the discrete time index.

The sequence of the given instantaneous power $p_{n}$ is limited by the (12) condition. It means that the source cannot provide current that transfers greater power than the source's maximum power $p_{n}^{M A X}$. In such case the source is delivering the adjustment current $i_{n}^{d}$, which transfers the maximum power $p_{n}^{M A X}$ at the moment. It will be shown in an example in Section 4.

\section{The solution of the optimal tasks for the multiphase source}

Skipping the discrete time index $n$ in (4), the $p^{M A X}$ task takes the form:

$$
\mathbf{i}^{T} \mathbf{r}_{0} \mathbf{i}-\mathbf{i}^{T} \mathbf{v} \rightarrow M I N
$$

Doing the same with (5), the $|\mathbf{i}|^{M I N}$ task takes the form:

$$
\mathbf{i}^{T}\left(\mathbf{1}+\lambda \mathbf{r}_{0}\right) \mathbf{i}-\lambda \mathbf{v}^{T} \mathbf{i} \longrightarrow M I N
$$

These minimum tasks were considered in paper [11] and its solving equations are:

$$
\mathbf{r}_{0} \mathbf{i}^{d}=\frac{1}{2} \mathbf{v}
$$

for the $p^{M A X}$ task, and:

$$
\left(\mathbf{1}+\lambda \mathbf{r}_{0}\right) \mathbf{i}^{\lambda}=\lambda \mathbf{r}_{0} \mathbf{i}^{d}
$$

for the $|\mathbf{i}|^{M I N}$ task. Solving (15) and (16) the multiphase vector of adjustment current is obtained:

$$
\mathbf{i}^{d}=\frac{1}{2} \mathbf{r}_{0}^{-1} \mathbf{v}
$$

and the $\lambda$ - based current vectors:

$$
\mathbf{i}^{\lambda}=\frac{1}{2}\left(\lambda^{-1} \mathbf{1}+\mathbf{r}_{0}\right)^{-1} \mathbf{v} .
$$

Simultaneously the source's power balance condition is met:

$$
\mathbf{i}^{T}\left(\mathbf{v}-\mathbf{r}_{0} \mathbf{i}\right)-p=0
$$

which allows to calculate the maximum power:

$$
p^{M A X}=\frac{1}{4} \mathbf{v}^{T} \mathbf{r}_{0}^{-1} \mathbf{v}
$$

The Lagrange's factor $\lambda$ in (17) can be calculated from the so-called power equation:

$$
F(\lambda)=P
$$

where

$$
F(\lambda)=\left(\mathbf{i}^{\lambda}\right)^{T}\left(\mathbf{v}-\mathbf{r}_{0} \mathbf{i}^{\lambda}\right)
$$

is the so-called source's energy function. That function can be approximated by expression [11]:

$$
F(\lambda)=\frac{2+\lambda r}{(1+\lambda r)^{2}} \lambda r p^{M A X},
$$

where

$$
r=\frac{\mathbf{v}^{T} \mathbf{v}}{\mathbf{v}^{T} \mathbf{r}_{0}^{-1} \mathbf{v}}
$$

is the so-called source normative resistance. Solving the energy equation by using the approximation function, the Lagrange's factor is obtained:

$$
\lambda_{*}^{-1}=r \frac{\sqrt{1-x}}{1-\sqrt{1-x}},
$$

where $x=\frac{p}{p^{M A X}}$ is fraction of the source's load. An instance of the optimal current is defined by (17) which now takes the form: 


$$
\mathbf{i}^{\text {opt }}=\frac{1}{2}\left(\lambda_{*}^{-1} \mathbf{1}+\mathbf{r}_{0}\right)^{-1} \mathbf{v}
$$

Introducing again the discrete time index causes appropriate values to take the sense of the instantaneous values. In this way, the sequential algorithm for determining the current's instantaneous-value vector is obtained:

$$
\left(\mathbf{i}_{n-1}, \mathbf{i}_{n-2}, \mathbf{i}_{n-3}, \ldots\right) \rightarrow \mathbf{i}_{n}
$$

which operates as follows:

- the instantaneous value of $\mathbf{v}_{n}$ is calculated:

$$
\mathbf{v}_{n}=\mathbf{e}_{n}-\sum_{m=1}^{\infty} \mathbf{z}_{m} \mathbf{i}_{n-m}
$$

- then the maximum instantaneous power:

$$
p_{n}^{M A X}=\frac{1}{4} \mathbf{v}_{n}^{T} \mathbf{r}_{0}^{-1} \mathbf{v}_{n}
$$

- next the instantaneous fraction of the source's load:

$$
x_{n}=\frac{p_{n}}{p_{n}^{M A X}},
$$

- next the source's normative-instantaneous resistance:

$$
r_{n}=\frac{\mathbf{v}_{n}^{T} \mathbf{v}_{n}}{\mathbf{v}_{n}^{T} \mathbf{r}_{0}^{-1} \mathbf{v}_{n}}
$$

- next the instantaneous Lagrange's factor:

$$
\lambda_{n}^{-1}= \begin{cases}r_{n} \frac{\sqrt{1-x_{n}}}{1-\sqrt{1-x_{n}}} & \text { for } x_{n}<1 \\ 0 & \text { for } x_{n}>1\end{cases}
$$

- and finally the instantaneous optimal current:

$$
\mathbf{i}^{\text {opt }}=\frac{1}{2}\left(\lambda_{n}^{-1} \mathbf{1}+\mathbf{r}_{0}\right)^{-1} \mathbf{v}_{n}
$$

\section{Examples for the single phase source}

Two similar examples are used for the single phase source in the discrete time domain. In both of them the source's internal impedance was assumed as follows:

$$
Z(s)=R+s L \underset{ }{\stackrel{s=f(1-z)}{\longrightarrow}} \stackrel{s f=1 / \tau}{\longrightarrow}+X_{L}-X_{L} z
$$

where: $R=4, X_{L}=f L=2$ (so-called digital reactance); from which the internal impedance sequence is obtained:

$$
\left\{z_{n}\right\}=\left\{R+X_{L} ;-X_{L} ; 0 ; 0 ; \ldots\right\}=\{6,-2,0,0, \ldots\} \text {, }
$$

thus: $z_{0}=r=6, z_{1}=-2$.
Also, in both cases, the constant sequence of the instantaneous power is assumed:

$$
\left\{p_{n}\right\}=\{200,200,200, \ldots\} \text {. }
$$

According to the internal impedance operator, voltage $v_{n}$ will take the form:

$$
v_{n}=e_{n}-z_{1} i_{n-1} .
$$

In Example 1 the source's non-sinusoidal voltage sequence is assumed as follows (Fig. 2):

$$
\left\{e_{n}\right\}=\left\{\begin{array}{l}
80,90,100,90,80,-80,-90,-100, \\
-90,-80,80,90,100,90,80,-80, \ldots
\end{array}\right\} .
$$

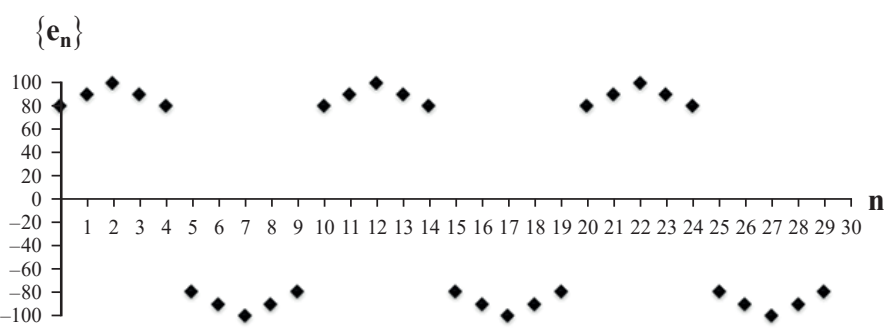

Fig. 2. The sequence of the source's voltage (Example 1)

The optimal online control algorithm begins with sample $n=0$ :

$$
\begin{gathered}
v_{0}=e_{0}-z_{1} i_{-1}=e_{0}=80, \\
i_{0}^{d}=\frac{v_{0}}{2 r}=6,666667, \\
p_{0}^{M A X}=\frac{\left(v_{0}\right)^{2}}{4 r}=266,666667, \\
x_{0}=\frac{p_{0}}{p_{0}^{M A X}}=0,75, \\
i_{0}=i_{0}^{d}\left(1-\sqrt{1-x_{0}}\right)=3,333333,
\end{gathered}
$$

then sample $n=1$ :

$$
\begin{gathered}
v_{1}=e_{1}-z_{1} i_{0}=96,666667, \\
i_{1}^{d}=\frac{v_{1}}{2 r}=8,055556, \\
p_{1}^{M A X}=\frac{\left(v_{1}\right)^{2}}{4 r}=389,351852, \\
x_{1}=\frac{p_{1}}{p_{1}^{M A X}}=0,513674, \\
i_{1}=i_{1}^{d}\left(1-\sqrt{1-x_{1}}\right)=2,437848,
\end{gathered}
$$




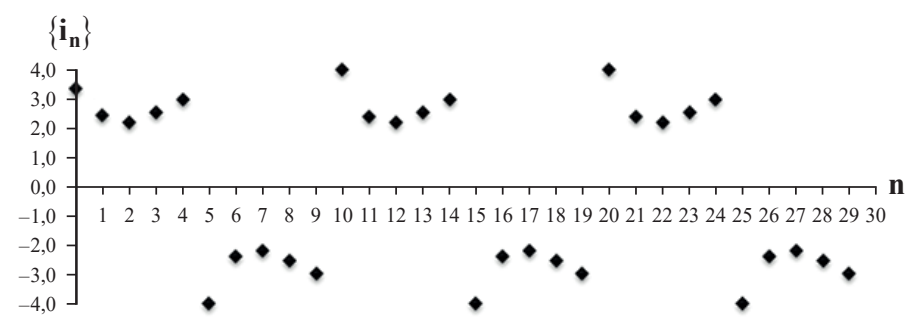

Fig. 3. The sequence of the optimal current (Example 1)

and so on. The optimal current $\left(i^{2}\right)^{M I N}$ is shown in Fig. 3.

In this example, all samples of current are optimal, which means that in each sample the required power $p_{n}$ is smaller than the maximum power $p_{n}^{M A X}$.

In Example 2, the source's voltage sequence is assumed as follows (Fig. 4):

$$
\left\{e_{n}\right\}=\left\{\begin{array}{l}
40,80,100,50,10,-40,-80,-100, \\
-50,-10,40,80,100,50,10, \ldots
\end{array}\right\} .
$$

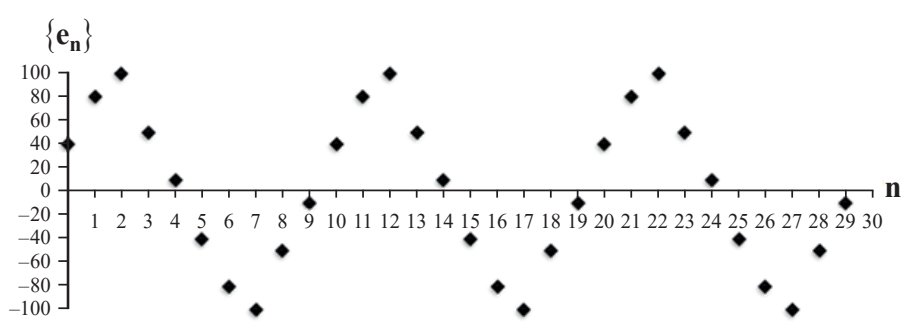

Fig. 4. The sequence of the source's voltage (Example 2)

The optimal online control algorithm for $n=0$ :

$$
\begin{gathered}
v_{0}=e_{0}-z_{1} i_{-1}=e_{0}=40, \\
i_{0}^{d}=\frac{v_{0}}{2 r}=3,333333, \\
p_{0}^{M A X}=\frac{\left(v_{0}\right)^{2}}{4 r}=66,666667, \\
x_{0}=\frac{p_{0}}{p_{0}^{M A X}}=3, \\
i_{0}=i_{0}^{d}=3,333333,
\end{gathered}
$$

and for $n=1$ :

$$
\begin{gathered}
v_{1}=e_{1}-z_{1} i_{0}=86,666667, \\
i_{1}^{d}=\frac{v_{1}}{2 r}=7,222222, \\
p_{1}^{M A X}=\frac{\left(v_{1}\right)^{2}}{4 r}=312,962963,
\end{gathered}
$$

$$
\begin{gathered}
x_{1}=\frac{p_{1}}{p_{1}^{\text {MAX }}}=0,639053, \\
i_{1}=i_{1}^{d}\left(1-\sqrt{1-x_{1}}\right)=2,883195
\end{gathered}
$$

and so on. The optimal current $\left(i^{2}\right)^{M I N}$ is shown in Fig. 5.

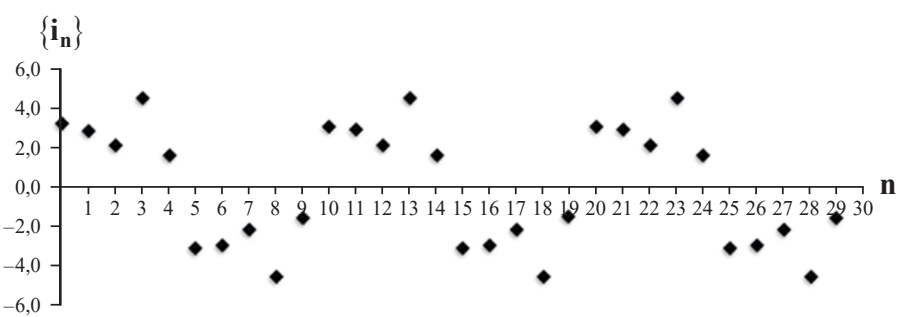

Fig. 5. The sequence of the optimal current (Example 2)

In sample $n=0$ it can be seen that the source's maximum power is lower than required $p_{0}$, therefore the optimal current is limited to the adjustment current (delivering the maximum power from the source) (12).

\section{Conclusion}

A new current control algorithm for the real-voltage source was formulated in the paper. The discrete time convolution voltage-current model with the internal impedance operator was used. First, the single-phase model was considered, then the multiphase one. The optimal source's current control brings to searching such a current signal as to provide both its minimum square value all the time and the given instantaneous power value. The control algorithm is recursive, i.e. the actual samples are obtained from the preceding ones. For the single-phase source, the problem of seeking current signal that provides the given instantaneous power has the unequivocal solution and thus, the obtained current signal has minimum square value all the time. The multiphase source works in a different way. The vector space of currents which deliver the given instantaneous power have infinite dimension and only one of them has the minimum RMS value.

It may be concluded that the obtained optimal current-control algorithm is a recursive digital filter with infinite recursion. However, in practice the infinite recursion can be approximately replaced by a finite one. Due to the stability of inner impedance operator, it can be assumed that

$$
\mathbf{z}_{m}=0
$$

(the zero matrix) for $m>M>0$. Then the recursion (18) takes the finite form:

$$
\mathbf{v}_{n}=\mathbf{e}_{n}-\sum_{m=1}^{\infty} \mathbf{z}_{m} \mathbf{i}_{n-m}
$$




\section{REFERENCES}

[1] M. Siwczyński and M. Jaraczewski, "The source optimal current calculation according to the instant power criterion", Przeglad Elektrotechniczny 85 (6), 155-158 (2009).

[2] M. Siwczyński and M. Jaraczewski, "The three-phase source optimal current calculation according to the instantaneous power exceed criterion", Przegląd Elektrotechniczny 85 (10), 71-75 (2009).

[3] M. Siwczyński and M. Jaraczewski, "Fractionally matched load to power source", Przeglad Elektrotechniczny 86 (4), 305-309 (2010) [in Polish].

[4] M. Siwczyński, S. Żaba, and A. Drwal, "Energy-optimal current distribution in a complex linear electrical network with pulse or periodic voltage and current signals. Optimal control”, Bull. Pol. Ac.: Tech. 64 (1), 45-50 (2016).

[5] M. Siwczyński and M. Jaraczewski, "Principle of similar equations for optimization of theory of electric power and energy", Przeglad Elektrotechniczny 86 (11a), 260-264 (2010) [in Polish].
[6] H. Akagi, Y. Kanazawa, and A. Nabae, "Generalized theory of the instantaneous reactive power in three-phase circuits", IPEC 83, 1375-1386 (1983).

[7] H. Akagi, E.H. Watanabe, and M. Aredes, Instantaneous Power Theory and Applications to Power Conditioning, Wiley-Interscience, A John Wiley and Sons, New Jersey, 2007.

[8] S. Sun and S. Huang, "On the meaning of nonsinusoidal active currents", IEEE Transactions on Instrumentation and Measurement 40 (1), 36-38 (1991).

[9] J.L. Willems, "A new interpretation of the Akagi-Nabae power components for nonsinusoidal three-phase situations", IEEE Transactions on Instrumentation and Measurement 41 (4), 523-527 (1992).

[10] F.Z. Peng and J.S. Lai, "Generalized instantaneous reactive power theory for three-phase power systems", IEEE Transactions on Instrumentation and Measurement 45 (1), 293-297 (1996).

[11] M. Siwczyński and K. Hawron, "Power theory optimization tasks - algorithms review", (to be published). 